\title{
Modelling wildlife species abundance using automated detections from drone surveillance
}

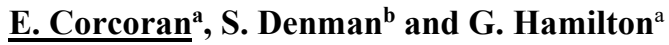 \\ ${ }^{a}$ School of Earth, Environmental and Biological Sciences, Queensland University of Technology, 2 George \\ Street, Brisbane, QLD, 4000, Australia. \\ ${ }^{b}$ School of Electrical Engineering and Computer Science, Queensland University of Technology, 2 George \\ Street, Brisbane, QLD, 4000, Australia \\ Email: g.hamilton@qut.edu.au
}

\begin{abstract}
Reliable estimates of abundance are critical to the conservation of threatened species. Aerial surveying is a sampling method that has been used to estimate wildlife abundance over large or inaccessible areas. An increasing trend in aerial surveying methodology is to use remotely piloted aircraft systems (RPAS), also known as drones, in lieu of traditional manned aircraft systems such as planes and helicopters. Studies which used RPAS instead of manned aircraft have recently attempted to analyse imagery using automated detection methods. While there are a number of advantages to this approach, there are also potential issues in abundance estimation using this data since the errors associated with using these approaches are largely unaccounted for in established models of abundance estimation.

In this paper we applied a model developed by Terletzky and Koons (2016) for fixed wing survey, to data derived from RPAS surveys that has been processed by an automated detection method for koalas. The data collected enabled ground-truthing of detections which allowed both the probability of detection and the probability of duplicate detection to be accounted for in abundance estimates, as well as a comparison between the estimates and the true number of koalas present on site.

Overall, it was found that the Terletzky \& Koons (2016) method resulted in artificial inflation of abundance estimates when using data collected from RPAS surveys with automated detection. This is likely to have resulted from false positive detections, which can have a considerable impact on the accuracy of automated wildlife counts. Incorporating more sources of error than the probability of detection and duplicate detection appears to be essential to improving abundance estimation for these novel survey methods. An exploration of additional covariates that could affect detection in RPAS-derived thermal imaging due the unique constraints of these technologies should be considered in future model development.
\end{abstract}

Keywords: Abundance modelling, wildlife detection, unmanned aerial vehicles (UAVS), machine learning 


\section{INTRODUCTION}

Abundance data are a strong predictor of extinction risk and are commonly used to determine conservation status (Renwick et al. 2012). This data is critical for effectively managing threatened species (Renwick et al. 2012). For some years, aerial sampling has been employed to gather estimates of wildlife abundance for species that occur over wide or inaccessible areas (Anderson \& Gaston 2013). More recently, there has been a shift towards remotely piloted aircraft systems (RPAS), commonly known as drones, to replace traditional manned aircraft such as planes or helicopters (Anderson \& Gaston 2013). An increasing number of these surveys have also incorporated automated or semi-automated detection methods in order to reduce the time taken to obtain wildlife counts and reduce the number of animals missed in surveys (Chretien et al. 2016; Hodgson et al. 2016; Seymour et al. 2017).

Many threatened species are in urgent need of efficient methods to regularly collect abundance data and inform the success of management actions. Koalas (Phascolarctos cinereus) are one such species that have been declared vulnerable throughout most of their range (McAlpine et al. 2015). There is therefore potential for koala management to benefit greatly from using RPAS surveys and automated detection methods to estimate abundance and inform species management. Unlike conventional aerial surveys for which the biases affecting abundance estimates have been extensively enumerated, the unique biases inherent in RPAS surveying of wildlife have rarely been investigated or accounted for in previous models of population numbers (Brack et al. 2018). The same is true of the biases uniquely affecting automated detection methods, despite many studies demonstrating that computer vision is not impacted by the same constraints as the human eye (Conn et al. 2014)

The aim of this paper is to investigate the feasibility of integrating automated detections of koalas in RPASderived thermal imaging into an existing method of abundance estimation for wildlife developed by Terletzky and Koons (2016). Their approach was determined to come the closest of any existing modelling technique to addressing the errors inherent in the method used to survey koalas in a study by Corcoran et al. (2019), as the number of missed animals and duplicate detections in surveys were calculated using telemetry to determine the presence and location of radio-collared animals within the survey area in a similar method to Terletzky \& Koons (2016). These biases were then factored into a final estimate of abundance for each survey.

\section{METHODS}

\subsection{Study Area and Survey Design}

The survey design and automated detection method used in this study are outlined in detail in Corcoran et al. (2019). In brief, two sites north and south of Petrie Mill, Queensland were selected to carry out RPAS surveys of the koala population. Collectively, these sites contained 48 radio-collared koalas that had been tracked extensively for several years by expert field ecologists (Waugh et al. 2016). The sites were also bordered by high-traffic roads and rivers leading to very low estimated immigration and emigration rates (Hanger et al. 2017). The presence of this isolated, individually tagged and traceable population therefore allowed for confirmation and enumeration of true positives results.

Data from five RPAS surveys at each site (ten total) conducted from May-August 2018 were used in development of abundance estimation models. All surveys were conducted at first light with a Matrice 600 Pro drone and A3 flight controller (DJ, Shenzhen, China). A FLIR Tau 2640 thermal camera (FLIR, Wilsonville, Oregon, United States of America) was mounted to the underside for image collection. The drone flew at a height of 60 metres above the ground, 30 metres above the tree canopy, at a speed of 8 metres per second in transects 20 metres apart encompassing the entirety of each site (Corcoran et al. 2019).

On the same day as RPAS surveying, ground surveys of the radio-collared koalas were conducted by expert field ecologists and the GPS co-ordinates of all koalas present were recorded. Data was simultaneously collected on the height of the trees koalas were found in, as well as the height of koalas in those trees, and each individual was assigned a visibility score from $0-5$. This score was based on how easily the koala could be visualised through the tree canopy, with 0 indicating the koalas was completely obscured and 5 indicating the koala was completely unobscured. 


\subsection{Automated Detection and Verification of Results}

An image detection algorithm (Corcoran et al. 2019) was then applied to the thermal images collected during the RPAS surveys using Python and an output of possible koala detections was generated along with their GPS co-ordinates. The locations of the radio-collared koalas were then matched to the detection coordinates to determine which koalas present were successfully identified, which were duplicate detections, and which had been missed.

\subsection{Models for Probability of Detection and Duplicate Detection}

Following the methodology of Terletzky and Koons (2016), generalized linear models (GLMs) with a binomial distribution and a logit link function were used to examine the impact of covariates on probability of detection ( $y=1$ for a successful detection, $y=0$ for a miss), and of a detection being a duplicate $(y=1$ when a detection is a duplicate, $\mathrm{y}=0$ for a unique detection). For probability of detection these covariates included visibility score, tree height and koala height in tree (Table 1). Group size and movement covariates suggested by Terletzky and Koons (2016) were not included as koalas tend to be solitary and remain stationary throughout surveying (Dique et al. 2003). However, the field of view of the drone led to overlap in images along and between transects, which could have resulted in the same koala being seen and detected multiple times during surveys. Therefore time since previous detection was considered a potential covariate for duplicate detection, as proposed by Terletzky and Koons (2016) (Table 1). Time since the beginning of the survey was not applicable to koalas as this was originally proposed to account for movement of animals during surveying which does not occur with this target species (Terletzky \& Koons 2016; Dique et al. 2003)

Table 1. List of covariates used in models of probability of detection and duplicate detection for automated detections of koalas in RPAS-derived thermal imagery.

\begin{tabular}{|l|l|l|}
\hline \multirow{2}{*}{ Model } & Covariate & Unit of Measurement \\
\hline Probability of Detection & Tree height & Metres $(\mathrm{m})$ \\
\cline { 2 - 3 } & Koala height in tree & Metres $(\mathrm{m})$ \\
\cline { 2 - 3 } & Visibility score & Likert scale $(0-5)$ \\
\hline Duplicate Detection & $\begin{array}{l}\text { Time since previous } \\
\text { detection }\end{array}$ & Seconds (sec) \\
\hline
\end{tabular}

Univariate GLMs were developed separately for probability of detection and probability of duplicate detection for each relevant covariate. These were ranked along with the null models according to Akaike Information Criterion (AIC), p-value, and reduction in residual deviance (Posada \& Buckley 2004). Generalized linear mixed models (GLMMs) incorporating the survey site as a random effect were also explored, however they failed to converge given the available data and thus inference was based on the simpler GLMs. The top performing models were also assessed with a Hosmer-Lemeshow test to examine them for evidence of poor fit (Hosmer \& Lemeshow 2000). All model development, selection and analysis was conducted using R statistical software including the 'Ime4', and 'ResourceSelection' packages (Bates et al. 2019; Lele et al. 2019; R Core 2018).

\subsection{Abundance Estimator}

Terletzky and Koons (2016) modified the Horvitz-Thompson (HT) abundance estimator based on corrections proposed by Marques et al. (2009) (Steinhorst \& Samuel 1989; Williams et al. 2002). This modified HT estimator was used to calculate individually-adjusted detection probabilities from the raw automated detection counts (1)

$$
\widehat{N}=\sum_{i=1}^{c} \frac{I_{i}\left(1-\hat{d}_{i}\right)}{\hat{p}_{i}}
$$

where $C$ is the number of counted individuals, $I_{i}$ is an indicator variable with the value of one for each counted individual in a survey, $d_{i}$ is the estimated probability of each counted individual being a duplicate, and $p_{i}$ the 
estimated probability of detection for each counted individual. The individual $p_{i}$ and $d_{i}$ variables are allowed to vary due to their respective covariates and $95 \%$ confidence intervals around the abundance estimates were created by first calculating the confidence interval as the fitted values of each GLM plus or minus two times the standard error on the link scale, and then using the inverse of the link function to map the fitted values, upper and lower limits back on to the response scale.

\section{RESULTS}

A mean of 16 individual radio-collared koalas were successfully detected by the algorithm at the north site with an average of 2 koalas missed in each survey. The number of radio-collared koalas detected at the south site was lower with a mean of 8 individuals successfully identified and 1 koalas missed in each survey. The number of duplicate detections was higher at the north site with a mean of 13 recorded in each survey compared to 4 duplicate detections found on average in south site surveys.

The mean visibility score for detected koalas $(2.4 \pm 0.81)$ was slightly higher than that of undetected koalas $(2.25 \pm 0.85)$. The height of trees that koalas were detected in $(17.95 \mathrm{~m} \pm 5.64)$ was higher on average than the mean height of trees inhabited by undetected koalas $(14.12 \mathrm{~m} \pm 5.97)$, and the mean height of koalas in trees was similar for both detected $(14.03 \mathrm{~m} \pm 4.83)$ and undetected $(14.12 \mathrm{~m} \pm 6.32)$ koalas. None of the covariates significantly explained variance in probability of detection (Table 2). Prior estimates of overall probability of detection from Corcoran et al. (2019) were incorporated into the final abundance estimates.

Table 2. Performance of univariate models of probability of automatically detecting koalas in RPAS-derived thermal imagery from surveys of Petrie Mill, Queensland in 2018.

\begin{tabular}{|l|l|l|l|l|}
\hline Model & P-value & Null Deviance & Residual Deviance & AIC \\
\hline Null Model & N/A & $\mathbf{1 5 1 . 6 3}$ & $\mathbf{1 5 1 . 6 3}$ & $\mathbf{1 5 3 . 6 3}$ \\
\hline Visibility Score & $\mathbf{0 . 3 6 7 7}$ & $\mathbf{1 5 1 . 6 3}$ & $\mathbf{1 5 0 . 8 1}$ & $\mathbf{1 5 4 . 8 1}$ \\
\hline Tree Height & $\mathbf{0 . 3 9 1 9}$ & $\mathbf{1 5 1 . 6 3}$ & $\mathbf{1 5 0 . 8 9}$ & $\mathbf{1 5 4 . 8 9}$ \\
\hline Koala Height & $\mathbf{0 . 9 2 6 4}$ & $\mathbf{1 5 1 . 6 3}$ & $\mathbf{1 5 1 . 5 6 2}$ & $\mathbf{1 5 5 . 6 2}$ \\
\hline
\end{tabular}

Time since previous detection explained $2.73 \%$ of the variance in the probability of an automated detection being a duplicate, which was found to be significant ( $\beta_{\text {Time since previous detection }}=-0.0012, \mathrm{SE}=0.0005464$, Table 3). This univariate model indicates that the likelihood of a detection being a duplicate decreased with a greater elapsed time since the previous detection was recorded (Figure 1). A Hosmer-Lemeshow test indicated there was no evidence of poor fit $(\chi$-squared $=8.404, \mathrm{df}=8, \mathrm{p}=0.395)$, and thus this model was incorporated into the modified HT abundance estimator (Hosmer \& Lemeshow 2000).

Table 3. Performance of univariate models of probability of recording a duplicate detection of a radiocollared koala in RPAS-derived thermal imagery from surveys of Petrie Mill, Queensland in 2018.

\begin{tabular}{|l|l|l|l|l|}
\hline Model & P-value & Null Deviance & Residual Deviance & AIC \\
\hline Null Model & N/A & $\mathbf{2 6 9 . 2 4}$ & $\mathbf{2 6 9 . 2 4}$ & $\mathbf{2 7 1 . 2 4}$ \\
\hline $\begin{array}{l}\text { Time Since } \\
\text { Previous } \\
\text { Detection }\end{array}$ & $\mathbf{0 . 0 2 9 1}$ & $\mathbf{2 6 9 . 2 4}$ & $\mathbf{2 6 1 . 8 8}$ & $\mathbf{2 6 5 . 8 8}$ \\
\hline
\end{tabular}




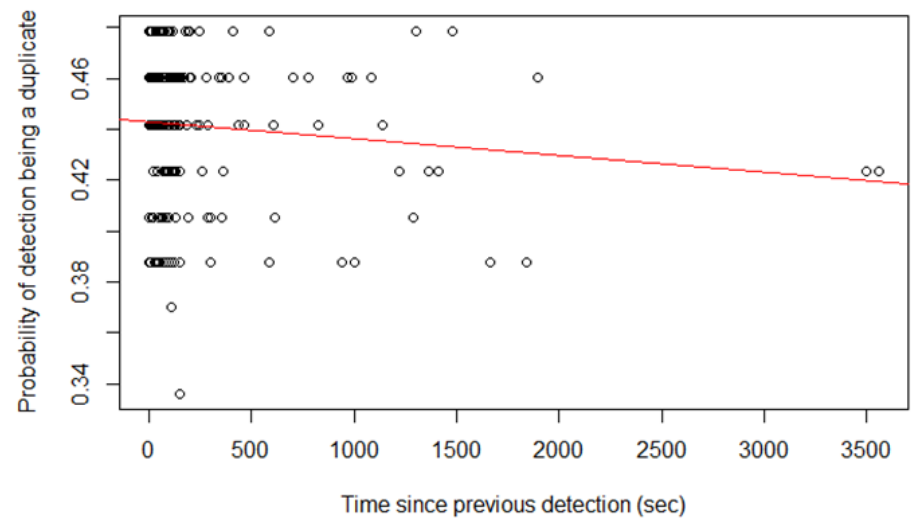

Figure 1. Predicted probability of a recorded detection of a radio-collared koala being a duplicate as a function of the elapsed time (in seconds) since the detection immediately prior was recorded by the algorithm.

The total number of automated detections including true positives, false positives, and duplicate detection was higher at the north site than the south site, with a mean of $45 \pm 15$ and $22 \pm 11$ detections per survey recorded at each site respectively (Table 4). At the north site, the true ground count of koalas present during surveying was only contained within the confidence interval generated by the modified HT estimator for one survey, May $22^{\text {nd }} 2018$ (Table 4). For the other four north site surveys both the abundance estimate and lower confidence interval were above the true count of koalas present (Table 4). This was the same for the south site, where the true count was only encapsulated by the confidence interval around the estimated abundance for the May $23^{\text {rd }}$ survey, with the lower confidence interval and abundance estimated higher than the actual number of koalas on site for all other surveys (Table 4).

Table 4. Estimated abundance ( $\pm 95 \%$ confidence intervals) of koalas at sites in north and south Petrie Mill, Queensland in 2018 compared to the raw number of detections recorded by the algorithm in each survey, and the true number of koalas present as determined by ground surveys of radio-collared individuals. True count (verified with telemetry) within the estimated confidence interval $(*)$.

\begin{tabular}{|l|l|l|l|}
\hline Survey & Raw Count & $\begin{array}{l}\text { Abundance Estimate } \pm 95 \% \\
\text { Confidence Interval }\end{array}$ & True Count \\
\hline May $22^{\text {nd }} 2018-$ North & $\mathbf{2 5}$ & $\mathbf{1 9}(\mathbf{1 5 , 2 2})^{*}$ & $\mathbf{2 0}$ \\
\hline June $12^{\text {th }} 2018-$ North & $\mathbf{6 3}$ & $\mathbf{3 5}(\mathbf{2 9}, \mathbf{4 1})$ & $\mathbf{2 0}$ \\
\hline July $10^{\text {th }} 2018-$ North & $\mathbf{4 1}$ & $\mathbf{2 4}(\mathbf{2 0 , 2 8})$ & $\mathbf{1 5}$ \\
\hline July $24^{\text {th }} 2018-$ North & $\mathbf{5 5}$ & $\mathbf{3 3}(\mathbf{3 0}, \mathbf{3 7})$ & $\mathbf{1 8}$ \\
\hline August $7^{\text {th }} 2018-$ North & $\mathbf{4 1}$ & $\mathbf{2 9}(\mathbf{2 4 , 3 3 )}$ & $\mathbf{1 9}$ \\
\hline May $23^{\text {rd }} 2018-$ South & $\mathbf{1 0}$ & $\mathbf{6 ( 5 , 7 ) ^ { * }}$ & $\mathbf{5}$ \\
\hline June $13^{\text {th }} 2018-$ South & $\mathbf{3 8}$ & $\mathbf{2 1 ( 1 8 , 2 4 )}$ & $\mathbf{1 1}$ \\
\hline July $11^{\text {th }} 2018-$ South & $\mathbf{1 7}$ & $\mathbf{1 3 ( 1 0 , 1 5 )}$ & $\mathbf{9}$ \\
\hline July $24^{\text {th }} 2018-$ South & $\mathbf{2 5}$ & $\mathbf{1 7}(\mathbf{1 5}, \mathbf{2 0})$ & $\mathbf{1 1}$ \\
\hline August $8^{\text {th }} 2018-$ South & $\mathbf{1 9}$ & $\mathbf{1 1}(\mathbf{9}, \mathbf{1 3})$ & $\mathbf{6}$ \\
\hline
\end{tabular}

\section{DISCUSSION AND CONCLUSIONS}

The abundance estimate and lower confidence interval obtained for the majority of surveys was greater than the actual number of target animals on site, and this result is similar to those of Terletzky and Koons (2016). However, unlike the results reported in that study the difference between the abundance estimates and true counts was not due to inflation of raw counts, but from a failure to reduce the initial number of detections (Terletzky \& Koons 2016). This suggests that accounting for duplicate detections alone may not be sufficient to eliminate spurious detections and obtain accurate abundance estimates from automated counts.

A logical next step in improving abundance estimations for automated detection methods is to account for other possible sources of error beyond probability of detection and rate of duplicate detection. These may include 
false detection rate, as misidentification of other animals or objects as the target species has been found to be a significant issue in automated wildlife counts; and availability error, which occurs when target individuals that are present in the survey area cannot be detected due to the limitations of the observer or sensor (Brack et al. 2018; Seymour et al. 2017). These sources of error could potentially be taken into account by groundtruthing false detections using telemetry or ground surveys, and gathering data on habitat conditions, such as canopy cover or tree density, which could impact visibility of target animals from RPAS footage (Brack et al. 2018). Weather conditions that may uniquely impact the image quality of RPAS-derived thermal imaging, such as wind and ambient temperature could also be considered as possible covariates modelling the probability of detection (Chabot \& Francis, 2016; Seymour et al., 2017). Unique aspects of RPAS survey design such as distance between transects and potential overlap in the field of view could also be investigated, as such conditions allow a single specimen to be viewed multiple times from multiple angles, which may impact the rate of duplicate detection, particularly in more mobile species (Brack et al. 2018).

\section{ACKNOWLEDGEMENTS}

The authors thank Endeavour Veterinary Ecology for their support in koala tracking, Moreton Bay Regional Council for access to field sites, and the HPC and Research Support Group at QUT for computational resources. This work was enabled by use of the Research Engineering Facility hosted by the Institute for Future Environments at QUT. E.C. was supported by an Australian Government Research Training Program Scholarship.

\section{REFERENCES}

Anderson, K. and Gaston, K. J. (2013). Lightweight unmanned aerial vehicles will revolutionize spatial ecology. Frontiers in Ecology and the Environment, 11, 138-146.

Bates, D., Maechler, M., Bolker, B., Walker, S., Christensen, R. H. B., Singmann, H., Dai, B., Scheipl, F., and Grothendick, G. (2019). Linear Mixed-Effects Models using 'Eigen' and S4. Version 0.21.

Brack, I. V., Kindel, A. and Oliveira, L. F. B. (2018). Detection errors in wildlife abundance estimates from Unmanned Aerial Systems (UAS) surveys: Synthesis, solutions, and challenges. Methods in Ecology and Evolution, 9, 1864-1873.

Chabot, D. and Francis, C. M. (2016). Computer-automated bird detection and counts in high-resolution aerial images: a review. Journal of Field Ornithology, 87, 343-359.

Chrétien, L.-P., Théau, J. and Ménard, P. (2016) Visible and thermal infrared remote sensing for the detection of white-tailed deer using an unmanned aerial system. Wildlife Society Bulletin, 40, 181-191.

Corcoran, E., Denman, S., Hanger, J., Wilson, B. and Hamilton, G. (2019). Automated detection of koalas using low-level aerial surveillance and machine learning. Scientific Reports, 9, 3208.

Conn, P. B., Ver Hoef, J. M., McClintock, B. T., Moreland, E. E., London, J. M., Cameron, M F., Dahle, S. P. and Boveng, P. L. (2014). Estimating multispecies abundance using automated detection systems: iceassociated seals in the Bering Sea. Methods in Ecology and Evolution, 5, 1280-1293.

Dique, D. S., Thompson, J., Preece, H. J., Villiers, D. L. d. \& Carrick, F. N. (2003). Dispersal patterns in a regional koala population in south-east Queensland. Wildlife Research, 30, 281-290

Hanger et al. (2017). Final Technical Report: Moreton Bay Rail Koala Management Program. Queensland State Government, Department of Transport and Main Roads, Brisbane.

Hodgson, J. C., Baylis, S. M., Mott, R., Herrod, A. and Clarke, R. H. (2016) Precision wildlife monitoring using unmanned aerial vehicles. Scientific Reports, 6, 22574.

Hosmer, D. W., and Lemeshow, S. (2000). Applied logistic regression. Second edition. John Wiley \& Sons, Hoboken, New Jersey, USA.

Lele, S. R., Keim, J. L. and Solymos, P. (2019). Resource Selection (Probability) Functions for UseAvailability

Data. Version 0.5.

McAlpine, C., Lunney, D., Melzer, C., Meckhorst, P., Phillips, S., Phalen, D., Ellis, W., Foley, W., Baxter, G., de Villiers, D., Kavangh, R, Adams-Hosking, C., Todd, C., Whisson, D., Molsher, R., Walter, M., Lawler, I. and Close, R. (2015). Conserving koalas: A review of the contrasting regional trends, outlooks and policy challenges. Biological Conservation, 192, 226-236. 
Marques, T. A., Thomas, L., Ward, J., DiMarzio, N. and Tyack, P. L. (2009). Estimating cetacean population density using fixed passive acoustic sensors: an example with Blainville's beaked whales. Journal of the Acoustical Society of America, 125, 1982-1994

Posada, D. and Buckley, T. R. (2004). Model selection and model averaging in phylogenetics: advantages of akaike information criterion and Bayesian approaches over likelihood ratio tests. Systematic Biology, 53, 793-808.

R Core Team (2018) R: A Language and Environment for Statistical Computing. R Foundation for Statistical Computing, Vienna.

Renwick, A. R. et al. (2012) Modelling changes in species' abundance in response to projected climate change. Diversity and Distributions, 18, 121-132.

Seymour, A. C., Dale, J., Hammill, M., Halpin, P. N. and Johnston, D. W. (2017). Automated detection and enumeration of marine wildlife using unmanned aircraft systems (UAS) and thermal imagery. Scientific reports, 7, 45127-45127.

Steinhorst, R. K., and Samuel, M. D. (1989). Sightability adjustment methods for aerial surveys of wildlife populations. Biometrics, 45, 415-425.

Terletzky, P. A., and Koons, D. N. (2016). Estimating Ungulate Abundance While Accounting for Multiple Sources of Observation Error. Wildlife Society Bulletin, 40, 525-536.

Waugh, C., Khan, S. A., Carver, S., Hanger, J., Loader, J., Polkinghorne, A., Beagley, K. and Timms, P. (2016). A Prototype Recombinant-Protein Based Chlamydia pecorum Vaccine Results in Reduced Chlamydial Burden and Less Clinical Disease in Free-Ranging Koalas (Phascolarctos cinereus). PLoS One, 11, e1046934.

Williams, B. K., Nichols, J. D. and Conroy, M. J. (2002). Analysis and management of animal populations. Academic Press, San Diego, California, USA. 